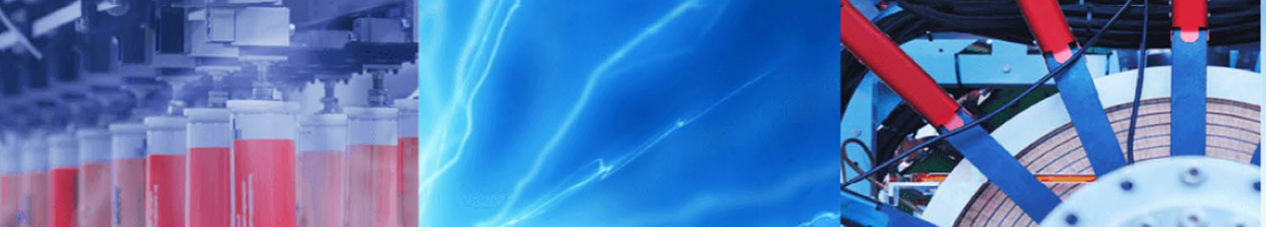

Research Article

\title{
Anchoring PNIPAM on ATP Surface via hydrogen bonding and coordination for a temperature-responsive adsorption of hydrophobic drug
}

\author{
Sheng Gong ${ }^{1} \cdot$ Tianhao He $^{1} \cdot$ Qizhang Huang ${ }^{1} \mathbb{D} \cdot$ Xugang Shu $^{1,3} \cdot$ Xinhua Zhou $^{1,2}$
}

Received: 21 November 2019 / Accepted: 30 March 2020 / Published online: 6 April 2020

(c) Springer Nature Switzerland AG 2020

\begin{abstract}
A thermo-responsive polymer, poly(N-isopropylacrylamide) (PNIPAM), was anchored on the surface of low-cost natural clay nanorods, attapulgite (ATP), through the interactions including hydrogen bonding and coordination. Premixing the monomers of NIPAM with ATP before polymerization caused that the obtained PNIPAM could distribute among the pore voids of ATP and function as a surface affinity conditioner in response to temperature change. The PNIPAM/ ATP composite was then applied in temperature-triggered adsorption and desorption of a hydrophobic drug (typically, an agricultural pesticide: acetamiprid). The results indicated a satisfactory adsorption capacity and reusability with the strong combination of PNIPAM and ATP. Furthermore, the adsorption mechanism was discussed, and the Sips model was employed to explain the adsorption behavior. It was proved to be beneficial for hydrophobic drug loading as well as its delivery, which may also have a potential to extend to other natural products and responsive polymers.
\end{abstract}

Keywords Adsorption · Thermo-responsivity $\cdot$ Poly $(n$-isopropylacrylamide) $\cdot$ Attapulgite $\cdot$ Hydrophobic drug

\section{Introduction}

Adsorption is regarded as an effective method for species separation and purification [1]. Adsorbate including drugs, metal ions, and the like can be a resource in some way. Besides, adsorbate retention on the active sites of adsorbent surface can decline its reusability. Thus, proper release of the adsorbate is desired for resource saving and reborn of the adsorbent. Release of the adsorbate can be modulated via the concept of "smart adsorbents" with pore voids for uptake and delivery of cargo molecules upon the responsive surface sites, which can be triggered by external stimulus including redox, $\mathrm{pH}$ value, and ionic strength contributing to chemical valves [2-4]. For instance, it was reported that polymers like alginate-chitosan were used as nanocapsules for controlled release of acetamiprid at different $\mathrm{pH}$ by responsive swelling [5]. Whereas to date, polymer textures with a reversible phase transition within the physiological temperature range are considered as the most promising responsive surfaces for external interaction, due to its convenient responsive operation and good reusability [6]. Especially, thermo-responsive polymers exhibit a temperature-dependent solution behavior that can change their surface hydrophilicity in response to a slight temperature change, and gain immerse attentions in responsive surface adsorption.

Electronic supplementary material The online version of this article (https://doi.org/10.1007/s42452-020-2643-1) contains supplementary material, which is available to authorized users.

Qizhang Huang, huangqz0305@163.com; $\bowtie$ Xugang Shu, xgshu@21cn.com | ${ }^{1}$ College of Chemistry and Chemical Engineering, Zhongkai University of Agriculture and Engineering, Guangzhou 510225, China. ${ }^{2}$ Guangzhou Key Laboratory of Efficient Utilization of Agrochemicals, Zhongkai University of Agriculture and Engineering, Guangzhou 510225, China. ${ }^{3}$ Guangdong Province Key Laboratory of Waterfowl Healthy Breeding, Zhongkai University of Agriculture and Engineering, Guangzhou 510225, China. 
Poly(N-isopropylacrylamide) (PNIPAM) with a lower critical solution temperature (LCST) around $32{ }^{\circ} \mathrm{C}$ represents one of the most popular thermo-responsive polymer surfaces for smart applications in optical film, hydrogel, cell attachment, micro-organism culture, and so on $[7,8]$. At temperature lower than LCST, PNIPAM is soluble in water, because its polar segments (carbonyl and amino) can form hydrogen bond with the polar-oriented water molecules around. On the contrary, at temperatures above LCST, the hydrogen bonds turn unstable so that PNIPAM is uneasy to be dissolved by water. Without surface hydrogen bonding, the nonpolar regions (alkyl groups) surrounded by reoriented water molecules display a clathrate-like structures, which was known as the hydrophobic effect of PNIPAM [9]. Also, PNIPAM undergoes a volume phase transition and attains the collapsed conformation of minimum size at above LCST, which can be further employed to alter the surface structure of pore voids $[6,10]$. Therefore, thermoresponsive PNIPAM is a competent candidate for preparing smart adsorbent by which the adsorbate molecules can be loaded or released through the equilibrium partitioning between the solution and polymer surfaces [11, 12].

Moreover, a facile tactic to smart adsorbent for molecule delivery can be based on anchoring stimuli-responsive polymers accessible to the pore voids of natural porous inorganic nanomaterials. Natural inorganic nanomaterials born with affluent porosity can potentially act as an adsorbent scaffold, having advantages of rich resource, low cost, and excellent durability [13]. For example, attapulgite (ATP) mines are abundant in nature, and quite inexpensive, which is a hydrous and porous magnesium-aluminum silicate fibrillar mineral with a molecular formula of $\left[\mathrm{Mg}_{5} \mathrm{Si}_{8} \mathrm{O}_{20}(\mathrm{OH})_{2}\left(\mathrm{OH}_{2}\right)_{4} \cdot 4 \mathrm{H}_{2} \mathrm{O}\right]$ [14]. With the aid of such nanoparticles like ATP, polymers can improve their thermal conductivity and mechanical stability dramatically $[15,16]$. Meanwhile, composites of thermo-responsive PNIPAM and inorganic nanoparticles can also receive amplified thermoresponsiveness by which PNIPAM can rapidly response to environmental temperature and finish molecular affinity transition, due to increased thermal conductivity by inorganic component [17].

On the other hand, the use of pesticides identified with toxicity and carcinogenicity will lead to chemical persistence in agricultural products and environmental problems, though it can protect the agricultural cultivation from insects and ensure mass production of food to feed the growing population [18, 19]. For example, acetamiprid [(E)-N1-[(6-chloro-3-pyridyl) methyl]-N2-cyano-N1-methylacetamidine, Fig. 1] with limited water solubility is a commonly-used active neonicotinoid insecticide against insects, which is now widely applied in crops, vegetables, fruits, and

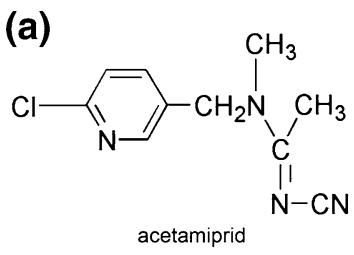

(b)<smiles>CCNC(=O)C(C)(C)CC(C)C(=O)NC(C)C</smiles>

Fig. 1 Molecular structures of a (E)-N1-[(6-chloro-3-pyridyl) methyl]-N2-cyano-N1-methylacetamidine and $\mathbf{b}$ poly(N-isopropylacrylamide)

tea all over the world [5]. However, its toxicity is also projecting a giant threat to human-being health and ecosystem. Thus, the release of such hazardous agrochemicals to food products and environment must be restricted. And the above-mentioned combination of ATP pore voids with PNIPAM hydrophobic effect may provide hydrophobic acetamiprid with an efficient temperature-responsive adsorption, and alleviate its harmful effect. With respect to the combination methods, inorganic nanomaterials incorporated with the polymers can be simply realized without covalent bonding, but facilely via physical blending or intercalation $[20,21]$. However, the blending mechanism and interaction between nanomaterial and polymer are not very clear, which are significant for anchoring polymer on inorganic surface for modulated affinity of an adsorbent. At the same time, the dispersion stability of rod-like ATP in polymers is a key problem for physical blending [22].

In present work, a facile and universal method to prepare smart adsorbent has been proposed through anchoring thermo-responsive PNIPAM on the low-cost natural clay nanorods of ATP. The monomers of NIPAM are pre-mixed with ATP aiming at a better intercalation before polymerization. Afterwards, the obtained PNIPAM is filled into the pore voids of ATP and functions as a surface affinity conditioner by temperature change. Consequently, the PNIPAM/ ATP composite displays a temperature-responsive adsorption for agricultural pesticide: acetamiprid, simultaneously with a favorable stability. Meanwhile, in this way, the natural nanorods of ATP can act as a structural strengthening agent, anchorage and cross-linker for the linear PNIPAM in solution (Scheme 1). The relevant surface interaction strengths are discussed and Sips model is employed to explain the temperature-responsive adsorption mechanism. The prepared composite is proved to be promising for reducing agricultural dependency on synthetic pesticides and dealing with pesticide residue problems, and this strategy may be extended to other natural products and responsive polymers. 


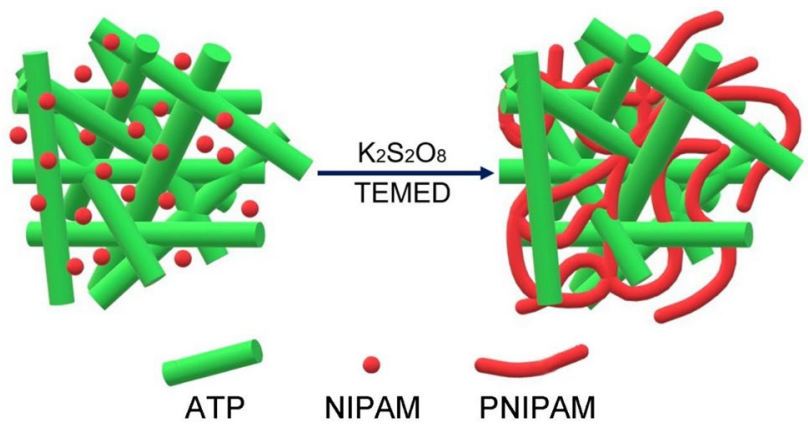

Scheme 1 Schematic Illustration of the Synthesis Procedure of the PNIPAM/ATP Nanocomposite

\section{Experimental methods}

\subsection{Materials}

$\mathrm{N}$-isopropylacrylamide (NIPAM) and N,N,N',N'-tetramethylethylenediamine (TEMED) were provided by Shanghai Aladdin Biochemical Technology Co., Ltd.. Potassium persulfate (KPS) and absolute ethanol (EtOH) were purchased from Tianjin Damao Chemical Co., Ltd. Deionized water was produced by a domestic equipment. All reagents used were of analytical-reagent grade.

\subsection{Synthesis of PNIPAM/ATP composite}

This synthesis procedure partially referred to the article [10]. Typically, $0.6 \mathrm{~g}$ of ATP mineral was first dispersed in a beaker with $20 \mathrm{~mL}$ of deionized water. The mixture was ultrasonically treated for $40 \mathrm{~min}$ for a good dispersion of ATP in water. Afterwards, $2 \mathrm{~g}$ of NIPAM monomers, $0.02 \mathrm{~g}$ of KPS as the oxidized initiator, and $50 \mu \mathrm{L}$ of TEMED as the accelerator were added to the above mixture with continuous stirring (Scheme 1). After polymerization and reaction for $7 \mathrm{~h}$ at room temperature, the obtained gel was washed by EtOH and dried in vacuum for a day to powders. The preparation of pure PNIPAM was conducted as the above procedure without adding ATP as well as any cross-linker.

\subsection{Quantum chemical calculations}

The quantum chemical calculations based on density functional theory (DFT) have been carried out using Gaussian 09 program package to predict the molecular orbitals, optimized geometry of molecular geometry, and Mulliken atomic charges at B3LYP functional and $6-31 \mathrm{G}(\mathrm{d}, \mathrm{p})$ basis set. Gauss-View 6.0 program is used for visualization of molecular orbitals and optimized geometry of molecule.

\subsection{Adsorption performance}

The adsorption thermodynamic studies were carried out by dispersing nanocomposite adsorbent powders (each dosage of $0.02 \mathrm{~g}$, marked as $\mathrm{m}$ ) in a $20 \mathrm{~mL}$ of acetamiprid solution (solvent: $\mathrm{EtOH}$ aqueous solution of $50 \mathrm{v} . \%$, the volume marked as $\mathrm{V}$ ) with different acetamiprid concentrations $\left(0.8,1.0,2.0,2.5\right.$ and $3 \mathrm{~g} / \mathrm{L}$, marked as $\left.C_{0}\right)$, respectively. After stirred at 25,35 and $45^{\circ} \mathrm{C}$ for $24 \mathrm{~h}$, the remained acetamiprid concentrations $\left(C_{\mathrm{e}}\right)$ in the solutions were used to calculate the equilibrium adsorption capacity $\left(q_{\mathrm{e}}=\left(C_{0}-C_{\mathrm{e}}\right) \cdot \mathrm{V} / \mathrm{m}\right)$. The obtained experimental data of $q_{\mathrm{e}}$ and $C_{\mathrm{e}}$ were analyzed with the Sips model, which shared both attributes of Langmuir and Freundlich models. It is defined as follow:

$q_{\mathrm{e}}=\frac{q_{\mathrm{m}} K_{\mathrm{s}} C_{\mathrm{e}}^{n_{\mathrm{s}}}}{1+K_{\mathrm{s}} C_{\mathrm{e}}^{n_{s}}}$

where $q_{\mathrm{m}}$ is the maximum adsorption capacity, $K_{\mathrm{s}}$ is the Sips isotherm constant related to the energy of adsorption, and $n_{\mathrm{s}}$ is the sorbent surface heterogeneity parameter. If the value of $n_{s}$ is 1 , the Sips model describes the typical Langmuir monolayer homogeneous adsorption behavior [23], otherwise the Sips model reflects a multilayer favorable adsorption condition $\left(n_{\mathrm{s}}>1\right)$. Besides, as the value of $C_{e}$ or $K_{s}$ is small enough (approaching 0 ), the Sips isotherm describes Freundlich isotherm behavior (a nonhomogeneous surface adsorption isotherm) [24].

\subsection{Characterization}

The morphologies of the ATP and PNIPAM/ATP were observed by field emission scanning electron microscopy (SEM, S-4800, operated at $5.0 \mathrm{kV}$ ). Infrared absorption spectra were recorded on a Fourier transform infrared (FT-IR) spectrometer (Thermo Fisher Scientific: Nicolet 6700) in the range of $400-4000 \mathrm{~cm}^{-1}$ with a resolution of $4 \mathrm{~cm}^{-1}$ (using $\mathrm{KBr}$ pellet). Thermogravimetric analysis (TGA) was performed with a Perkin-Elmer TGA-7 system at a scan rate of $10{ }^{\circ} \mathrm{C} \mathrm{min}^{-1}$ and a $\mathrm{N}_{2}$ flow of $20 \mathrm{~mL} \mathrm{~min}^{-1}$. The phase transitions were measured by a differential scanning calorimeter (DSC) microcalorimeter (MicroCal, Inc.) at a heating rate of $10{ }^{\circ} \mathrm{C} \mathrm{min}^{-1}$. Absorbances of the solutions were examined by UV-VISNIR spectrophotometer (Lambda750, USA Perkin Elmer Company). 
Fig. 2 SEM images of a pure ATP and $\mathbf{b}$ the prepared PNIPAM/ATP nanocomposites (the insets are amplification of local areas)
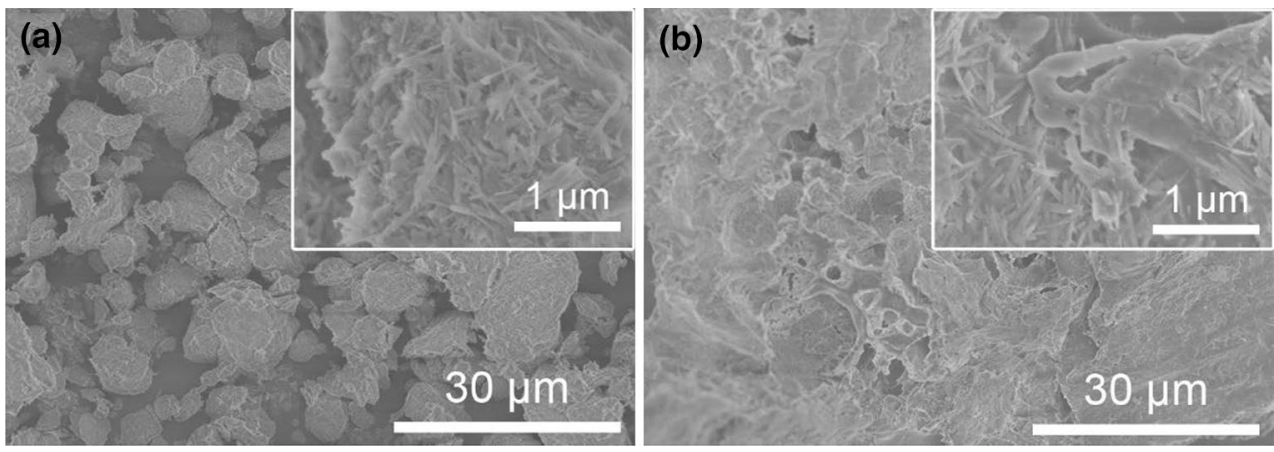

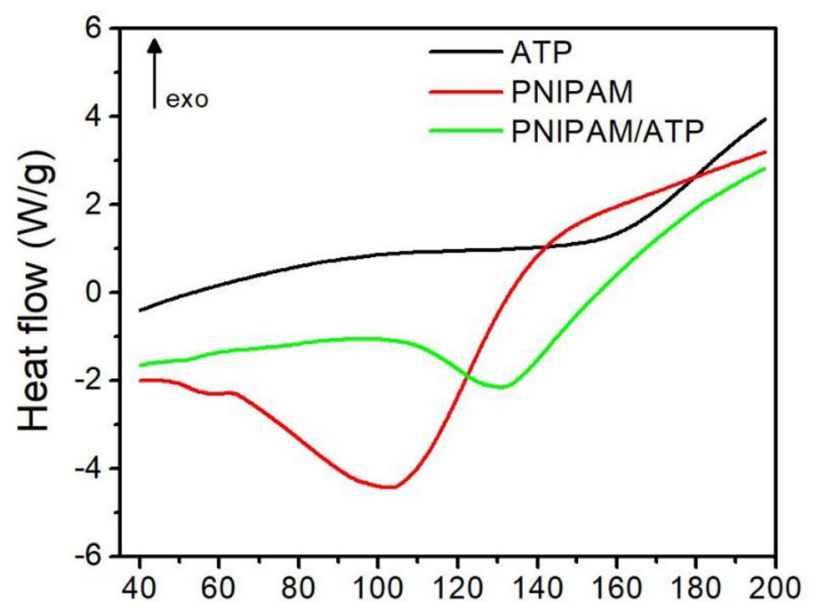

Fig. 3 DSC curves under $\mathrm{N}_{2}$ for pure ATP, PNIPAM, and the PNIPAM/ ATP nanocomposite

\section{Results and discussion}

The preparation procedure of anchoring PNIPAM on ATP is illustrated in Scheme 1. ATP is a kind of natural mine with inner-connected nano-pore voids that are created by the spatial stacking of ATP rods. Premixing of the monomer NIPAM with ATP can benefit the access of the obtained polymer to the pore voids due to the easier migration of monomers. As a result, after polymerization, the final chain-like PNIPAM is likely to go throughout the inner-connected pore voids and distributes along the pore canals, which depends on a strong combination of PNIPAM and ATP clay. Such presuppose of the strong combination in Scheme 1 is firstly verified by typical field emission scanning electron microscopy (SEM) images (Fig. 2). Figure 2a shows that the agglomerates of ATP nanorods produce particles in size of $\sim 10 \mu \mathrm{m}$. With amplified view, the inset reflects that ATP nanorods have a diameter of dozens $\mathrm{nm}$ and a length of $200-1000 \mathrm{~nm}$. The stacking of ATP nanorods create pore voids of dozens $\mathrm{nm}$. After polymerization of NIPAM previously intercalated in ATP, the pore voids in ATP can be filled by

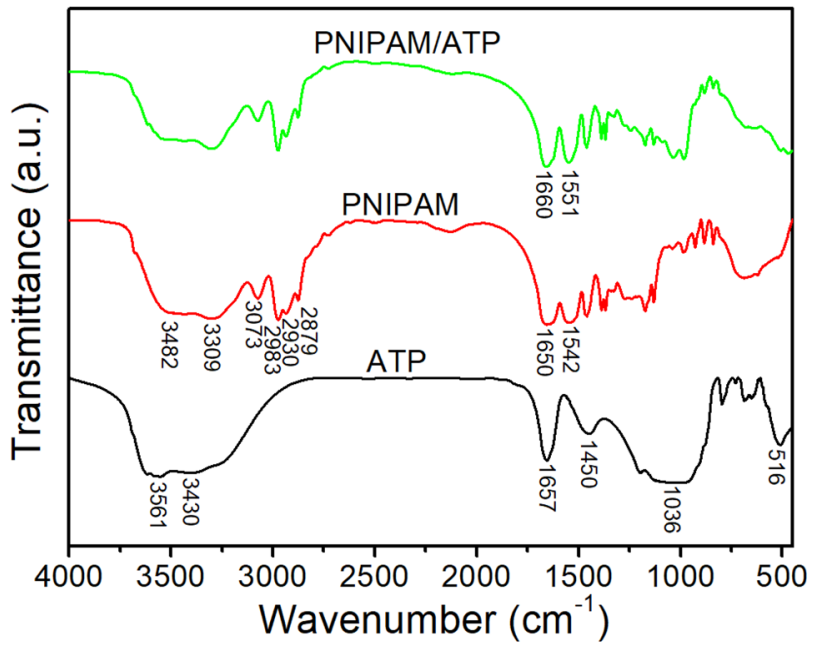

Fig. 4 FTIR spectra of pure ATP, PNIPAM, and the PNIPAM/ATP nanocomposite

PNIPAM (in Fig. $2 b$ and the inset). This result indicates a compatible mixing of PNIPAM and ATP.

The interaction between PNIPAM and ATP is verified by DSC techniques. Figure 3 depicts DSC curves of ATP, PNIAM, and PNIPAM/ATP composites with increasing temperature. For pure ATP, a broad endothermic peak appears in the temperature range of $100-200^{\circ} \mathrm{C}$, which can be attributed to the desorption of free and structural water. For PNIPAM, an endothermic peak located around $105^{\circ} \mathrm{C}$ may be due to the phase transition of melting. However, when complexed with ATP, the melting point increases to $130^{\circ} \mathrm{C}$, implying the surface interaction (hydrogen bond and coordination) between PNIPAM and ATP. In accordance with the above SEM observation, DSC analysis again argues the access of PNIPAM to the pore voids of ATP via surface interaction.

To explore the surface interaction, FTIR spectroscopy is carried out (Fig. 4). In the case of pure ATP, the absorption bands at 3561 and $3430 \mathrm{~cm}^{-1}$ are assigned to the $\mathrm{O}-\mathrm{H}$ stretching vibrations of free water and structural water molecules (including zeolitic and adsorbed water) [25]. 
The adsorption peaks at about 1657 and $1450 \mathrm{~cm}^{-1}$ are ascribed to the stretching of $\mathrm{C}=\mathrm{O}$ and $\mathrm{C}-\mathrm{O}$, which may be resulted from the impurity of carbonate [26]. The deformation vibration of $\mathrm{O}-\mathrm{H}$ on ATP surface may appear at about $1450 \mathrm{~cm}^{-1}$. The bands located at 1036 and $516 \mathrm{~cm}^{-1}$ possibly derive from the stretching vibration of $\mathrm{Si}-\mathrm{O}-\mathrm{Si}$ and $\delta_{\mathrm{Si}-\mathrm{O}}$ [27]. In terms of PNIPAM, the bands at 3482 and $3309 \mathrm{~cm}^{-1}$ are attributed to $\mathrm{N}-\mathrm{H}$ stretching. In addition, it shows two characteristic peaks for PNIPAM at about 1650 (carbonyl and amide stretching) and $1542 \mathrm{~cm}^{-1}$ (N-H stretching) [28]. In the range of $2860-3100 \mathrm{~cm}^{-1}$, the absorption bands represent the $\mathrm{C}-\mathrm{H}$ vibrations respectively at $3073\left(-\mathrm{CH}_{3}\right), 2983(-\mathrm{CH}-), 2930$ and $2879 \mathrm{~cm}^{-1}$ $\left(-\mathrm{CH}_{2}\right)$ [29]. For PNIPAM/ATP composites, the bands centered at 1650 and $1542 \mathrm{~cm}^{-1}$ (carbonyl and amide vibration) move to 1660 and $1551 \mathrm{~cm}^{-1}$, respectively, indicating that the amide section of PNIPAM probably have hydrogen bonds with the hydroxyls on ATP clay surface, or have coordination with the exposed $\mathrm{Mg}^{2+}$ on ATP facets.

In order to further confirm the surface interaction sites and mechanisms, quantum chemical calculations based on DFT (see experimental) are used for visualization of the molecular orbitals and optimized geometry, and the values of Fukui functions. Molecular orbital plots of PNIPAM (polymerization degree, $n=1$ ) are shown in Fig. 5a for observing chemical reactivity of the atoms on repeating unit. The vicinal orbitals of HOMO and LUMO mainly distribute within the area around amide $(\mathrm{O}=\mathrm{C}-\mathrm{N})$, playing a key role of electron donor and acceptor, respectively. Fukui functions $\left(f_{k}^{+}, f_{k}^{-}\right)$describe the (a)

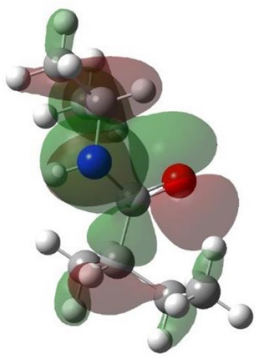

HOMO

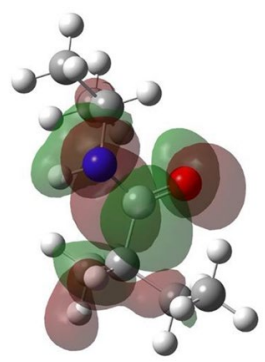

LUMO (b)

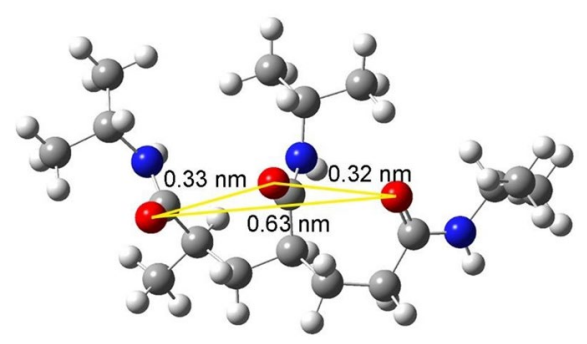

Fig. 5 Visualization of DFT calculation of a PNIPAM $(n=1)$ molecular orbitals (HOMO, LUMO), and $\mathbf{b}$ the optimized PNIPAM $(n=3)$ geometry labeled with $\mathrm{O}$ atomic distance nucleophilic and electrophilic ability of the atoms, using Mulliken atomic charges given in Table S1. In accordance with the above orbital distribution, $\mathrm{O}$ and $\mathrm{N}$ atoms have the greatest ${f_{k}}^{+}$and $f_{k}^{-}$, denoting the interaction sites of amide. However, $\mathrm{O}$ atom with greater Fukui functions $\left(f_{k}{ }^{+}=0.2501, f_{k}^{-}=0.2140\right)$ has higher chemical reactivity (higher nucleophilic and electrophilic possibility) than $\mathrm{N}$ atom (with $f_{k}^{+}=0.1688, f_{k}^{-}=0.0583$ ) [30]. Thus, the interaction sites of PNIPAM with ATP surface should be mainly ascribed to the carbonyl $(\mathrm{O}=\mathrm{C})$ that may have hydrogen bonding and coordination with the ATP surface. On the other side, the optimized molecular geometry of PNIPAM $(n=3)$ is obtained through the same calculation method (Fig. 5b). The distance between two adjacent $\mathrm{O}$ atoms of $\mathrm{O}=\mathrm{C}$ is equal to $0.33 \mathrm{~nm}$, approximately, while the interval of three $O$ atoms is about $0.63 \mathrm{~nm}$. It is reported that the lattice spacing of (002) plane on ATP clay is close to $0.65 \mathrm{~nm}$ (according to Bragg equation) [31], revealing a distance match between three $\mathrm{O}$ atomic interval and the (002) plane spacing. As a result, the nucleophilic carbonyl $(\mathrm{O}=\mathrm{C})$ is easy to coordinate with $\mathrm{Mg}^{2+}$ on (002) plane exposed to the ATP surface. In general, the surface interaction mechanism of anchoring PNIPAM on ATP can be interpreted by hydrogen bonding of carbonyl or amino with the ATP hydroxy, and carbonyl coordinating with $\mathrm{Mg}^{2+}$ on the natural clay plane.

The thermal stability of the PNIPAM/ATP composites is investigated by thermal gravimetric analysis (TGA). The mass loss curves for ATP, PNIPAM, PNIPAM/ATP are shown in Fig. 6. For pure ATP, the tiny weight losses below $120^{\circ} \mathrm{C}$ and at around $200{ }^{\circ} \mathrm{C}$ can be attributed to the release of free water and the structural water of ATP, respectively [32]. For the PNIPAM/ATP composites, similar to that of ATP, the main weight loss below $120^{\circ} \mathrm{C}$ can be assigned to the loss of free water, and, in the range of 150 and $200^{\circ} \mathrm{C}$, to the loss of the structural water. The weight of PNIPAM/

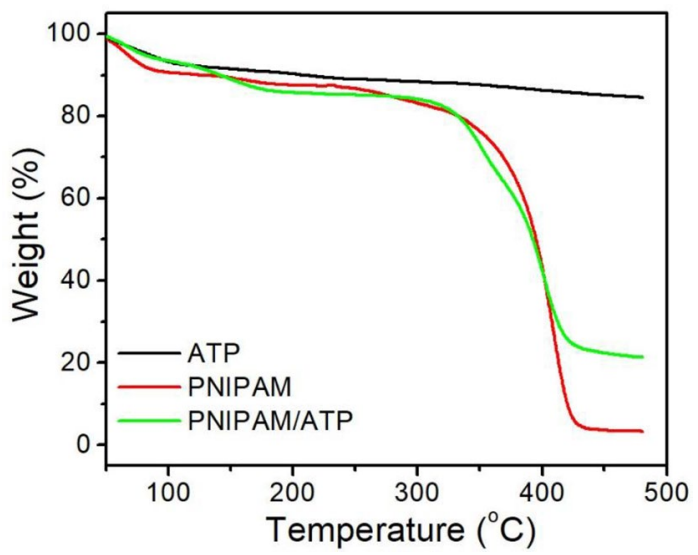

Fig. 6 TGA curves under $\mathrm{N}_{2}$ for pure ATP, PNIPAM, and the PNIPAM/ ATP nanocomposite 
ATP composites remain almost constant between 200 and $340^{\circ} \mathrm{C}$, but show a sudden decrease above $340^{\circ} \mathrm{C}$ which can be attributed to the decomposition of PNIPAM (the same as that of pure PNIPAM). Eventually, the residual weight of PNIPAM/ATP holds a proportion of $23 \mathrm{wt} \%$ as calculated from TGA analysis. The result further reveals that the proportion of ATP clay in the prepared PNIPAM/ATP composites is approximately $23 \mathrm{wt} \%$.

The adsorption abilities of ATP, PNIPAM, and PNIPAM/ ATP composite towards acetamiprid are evaluated by progressive contact of the above adsorbents (in powder state, each dosage of $0.02 \mathrm{~g}$ ) with $20 \mathrm{~mL}$ of acetamiprid solution (concentration: $3 \mathrm{~g} / \mathrm{L}$, solvent: EtOH/water $50 \mathrm{v} . \%$ ) for $24 \mathrm{~h}$ at $25^{\circ} \mathrm{C}$. Their adsorption capacities are exhibited in Fig. 7 . In this condition, natural ATP with surface hydroxyl has the smallest adsorption capacity of $94.24 \mathrm{mg} / \mathrm{g}$, in spite of a number of pore voids inside. In comparison, due to the limited solubility of acetamiprid in water, PNIPAM with alkyl on the main carbon chain has a better surface affinity to hydrophobic acetamiprid and thus a much greater adsorption capacity of $245.48 \mathrm{mg} / \mathrm{g}$. When incorporated with ATP, PNIPAM molecule has hydrogen bonds and coordination with the surface hydroxyls and $\mathrm{Mg}^{2+}$ on ATP, which chemically modified the inner wall of pore voids and improved the adsorption capacity of ATP to $185.46 \mathrm{mg} / \mathrm{g}$.

Surface affinity plays a key role in adsorption. The LCST of both PNIPAM and PNIPAM/ATP composite are determined by water contact angle versus temperature (S2), reflecting their LCST dwelling at about $32.1^{\circ} \mathrm{C}$ (Figure S2). Therefore, around $32.1{ }^{\circ} \mathrm{C}$, the surface affinity of PNIPAM/ ATP composite can be modulated by its hydrophobic effect. Meanwhile, PNIPAM compatibly mixed with inorganic nanomaterials (ATP) will receive amplified thermoresponsivity [17], thus the surface affinity of PNIPAM/ATP composite can be sensitive and rapidly responsive to temperature change.

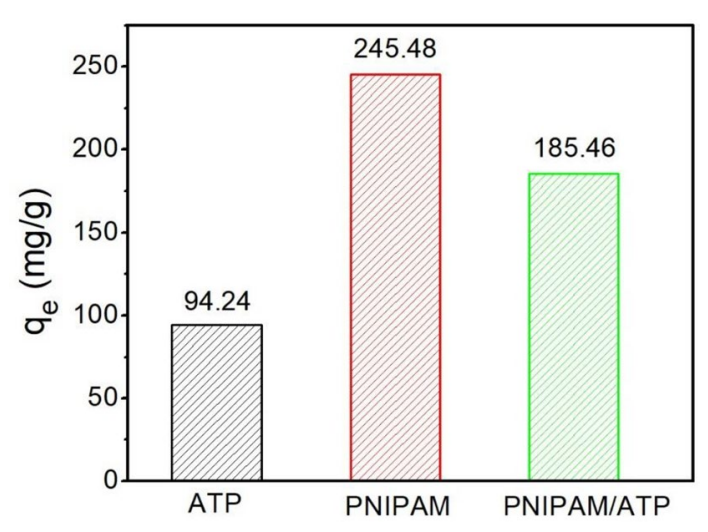

Fig. 7 Adsorption of acetamiprid ( $3 \mathrm{~g} / \mathrm{L}$ ) by pure ATP, PNIPAM, and PNIPAM/ATP nanocomposite in EtOH solution (50 v.\%) for $24 \mathrm{~h}$ at $25^{\circ} \mathrm{C}$

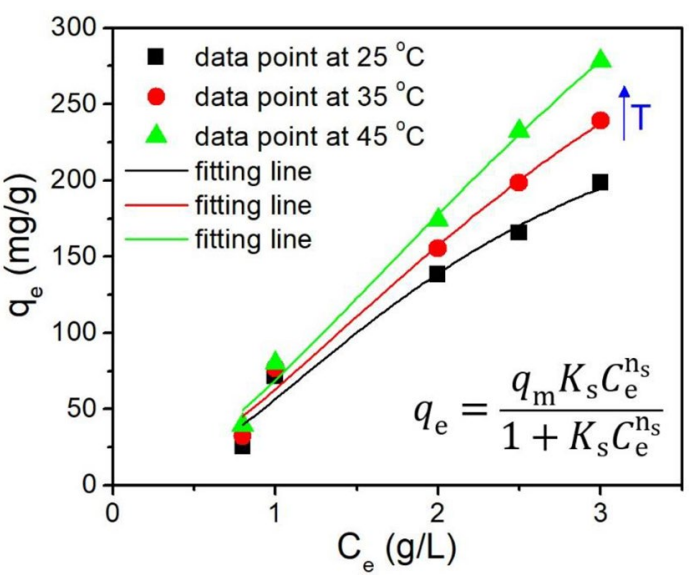

Fig. 8 Isotherm sorption of acetamiprid by PNIPAM/ATP nanocomposite in EtOH solution (50 v.\%) at different temperature

The effect of temperature on the adsorption behavior of PNIPAM/ATP composite is continued to be explored in Fig. 8. The relationship between the residual amount $\left(C_{\mathrm{e}}\right)$ of acetamiprid and the adsorbed acetamiprid amount per unit mass of adsorbent $\left(q_{\mathrm{e}}\right)$ at different temperature $\left(25,35\right.$, and $\left.45^{\circ} \mathrm{C}\right)$ are well fitted by the Sips model with correlation coefficient $R^{2}$ of $0.95,0.97$, and 0.99 , respectively. Listed in Table 1, the fitted value of the Sips isotherm adsorption constant $\left(K_{s}\right)$ and heterogeneity $\left(n_{s}\right)$ decrease with increasing temperature. The $n_{s}$ in the Sips equation represents the heterogeneity of adsorbent surface, and the $n_{s}$ value being closer to 1 indicates that the adsorbent surface is more homogeneous [33]. At higher temperature, the molecule collapse of PNIPAM with the hydrophobic effect results in the ATP surface sites wrapped and the pore voids packed by PNIPAM, leading to a more homogeneous adsorption surface. On the other hand, $K_{s}$ is positively correlated to the adsorption strength [33], revealing that higher temperature may intensify the thermal motion and thus weaken the interactions (van der waals force) between acetamiprid and the adsorbent. However, regardless of the weakened adsorption strength, hydrophobic effect of PNIPAM beyond LCST render PNIPAM/ ATP composite with lower polar affinity [9]. Consequently, low-polar PNIPAM/ATP composite can accumulate more

Table 1 Fitted parameters of Sips model isotherm adsorption behavior of PNIPAM/ATP composite towards acetamiprid at 25, 35, and $45^{\circ} \mathrm{C}$

\begin{tabular}{lllll}
\hline $\begin{array}{l}\text { Temperature } \\
\left({ }^{\circ} \mathrm{C}\right)\end{array}$ & $q_{\mathrm{m}}(\mathrm{mg} / \mathrm{g})$ & $K_{\mathrm{s}}$ & $n_{\mathrm{s}}$ & $\mathrm{R}^{2}$ \\
\hline 25 & 298.22 & 0.23 & 1.90 & 0.95 \\
35 & 503.61 & 0.14 & 1.67 & 0.97 \\
45 & 698.89 & 0.11 & 1.64 & 0.99 \\
\hline
\end{tabular}



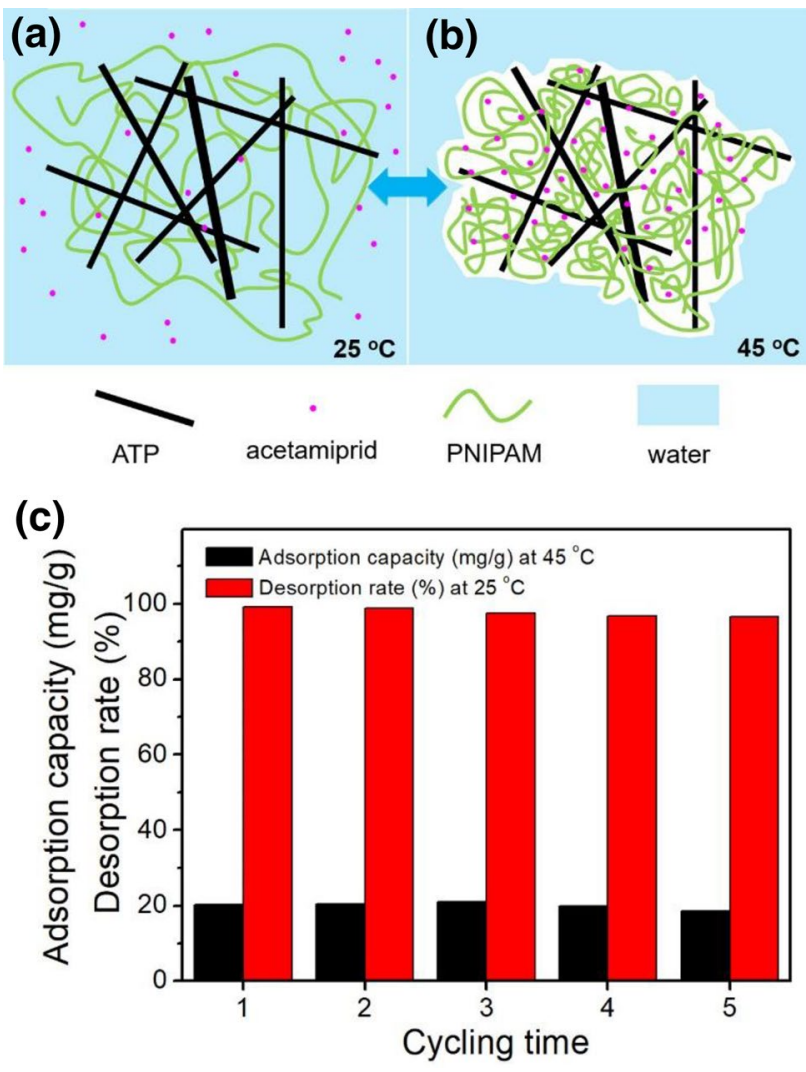

Fig. 9 Temperature-controlled adsorption of acetamiprid in water by PNIPAM/ATP composite: adsorption mechanism illustration at a 25 and $\mathbf{b} 45^{\circ} \mathrm{C}$, and $\mathbf{c}$ reversibility test

acetamiprid (with hydrophobic groups like phenyl, methyl, and methylene) from EtOH solution, which accounts for the largest fitted maximum adsorption capacity $q_{m}$ of PNIPAM/ATP composite at $45^{\circ} \mathrm{C}$.

When in water, hydrogen bonds between PNIPAM polar groups and water molecules are the main reason for polymer dissolution. Temperature beneath LCST facilitates the formation of hydrogen bonds with the exothermic formation enthalpy, but not beyond LCST. In addition, acetamiprid has a limited solubility in water. Thus, the hydrophobic effect of PNIPAM in water is more obvious for the adsorption of acetamiprid than in EtOH solution. At $25^{\circ} \mathrm{C}$, water-compatible PNIPAM/ATP composite is expected to have close affinity to water, leading to no distinguished distribution preference of acetamiprid (Fig. 9a). But at $45{ }^{\circ} \mathrm{C}$, hydrophobic effect may cause phase separation and precipitation, where polymer-water contacts are replaced by polymer-polymer and water-water contacts [34]. In this case, acetamiprid tends to enrich in PNIPAM/ATP composite from water (Fig. 9b). This mechanism can be applied in temperaturecontrolled release of acetamiprid in water, dealing with excessive pesticide contamination. For this purpose, pure water is used as the solvent instead of the above EtOH solution, aiming at simulation of natural environment. Besides, $0.5 \mathrm{~g}$ of the composite as adsorbent are dispersed in acetamiprid solution $(30 \mathrm{~mL}, 0.6 \mathrm{~g} / \mathrm{L}$ in water) with temperature switch at $45^{\circ} \mathrm{C}$ (beyond LCST) for 24-h adsorption and at $25^{\circ} \mathrm{C}$ (beneath LCST) for $24-\mathrm{h}$ desorption. As shown in Fig. 9c, after 5 times of adsorption/desorption cycles, above $95 \%$ adsorption capacity and the corresponding desorption rate are remained, compared with those of the first cycle. The result indicates that the PNIPAM/ATP composite performs a good reversibility with satisfactory stability.

\section{Conclusions}

In summary, a temperature-responsive smart adsorbent of PNIPAM/ATP composite has been prepared by anchoring PNIPAM on the surface of ATP clay. Pre-mixing of NIPAM with natural ATP before polymerization results in a good interpenetration between the obtained PNIPAM and ATP, where the PNIPAM distributes along the pore canals in ATP, due to the amide interaction of hydrogen bonding with the hydroxyls and coordination with $\mathrm{Mg}^{2+}$ on ATP surface. An ideal blend renders the PNIPAM/ATP composite with excellent stability and amplified thermoresponsivity. Thus, the PNIPAM/ATP composite presents an outstanding temperature-responsive adsorption of acetamiprid with a considerable reversibility, where adsorption and desorption can be modulated by the temperature switch of 45 and $25^{\circ} \mathrm{C}$. The adsorption behavior is well fitted by Sips model, and hydrophobic effect of PNIPAM is responsible for the equilibrium partitioning of acetamiprid from solution. Last but not the least, the surface interaction between natural clay products and responsive polymer can be regarded as a low-cost and convenient tactic for smart adsorption in future applications.

Acknowledgements This work is supported by Natural Science Foundation of Guangdong Province (2018A0303130068), Guangdong Science and Technology Department (2018050506081), and Guangdong graduate education innovation program (2015-2). Especially, sincere thanks for her selfless dedication and warm care from Miss Shen, Jingli.

\section{Compliance with ethical standards}

Conflict of interest No conflict of interest exits in this manuscript, and manuscript is approved by all authors for publication. Moreover, the work described was original research that has not been published previously, and not under consideration for publication elsewhere, in whole or in part. All the authors listed have approved the manuscript that is enclosed. 


\section{References}

1. Dong YY, Liu S, Liu Y-J, Meng L-Y, Ma M-G (2017) Ag@Fe304@ cellulose nanocrystals nanocomposites: microwave-assisted hydrothermal synthesis, antimicrobial properties, and good adsorption of dye solution. J Mater Sci 52:8219

2. Nguyen TD, Liu Y, Saha S, Leung KC, Stoddart JF, Zink JI (2007) Design and optimization of molecular nanovalves based on redox-switchable bistable rotaxanes. J Am Chem Soc 129:626

3. Leung CF, Nguyen TD, Stoddart JF, Zink JI (2006) Supramolecular nanovalves controlled by proton abstraction and competitive binding. Chem Mater 18:5919

4. Casasús R, Climent E, Marcos MD et al (2008) Dual aperture control on $\mathrm{pH}$ - and anion-driven supramolecular nanoscopic hybrid gate-like ensembles. J Am Chem Soc 130:1903

5. Kumar S, Chauhan N, Gopal M, Kumar R, Dilbaghi N (2015) Development and evaluation of alginate-chitosan nanocapsules for controlled release of acetamiprid. Int J Biol Macromol 81:631

6. Schmidt S, Zeiser M, Hellweg T, Duschl C, Fery A, Möhwald H (2010) Temperature-responsive substrates: adhesion and mechanical properties of PNIPAM microgel films and their potential use as switchable cell culture substrates. Adv Funct Mater 20:3235

7. Matthias K, Isabel PS, Jorge PJ, Thomas H, Liz-Marzán LM (2010) Nanorod-coated PNIPAM microgels: thermoresponsive optical properties. Small 3:1222

8. Schmaljohann D, Oswald J, Jørgensen B, Nitschke M, Beyerlein D, Werner C (2003) Thermo-responsive PNiPAAm-g-PEG films for controlled cell detachment. Biomacromol 4:1733

9. Schild HG (1992) Poly (N-isopropylacrylamide): experiment, theory and application. Prog Polym Sci 17:163

10. Otake K, Inomata H, Konno M, Saito S (1990) Thermal analysis of the volume phase transition with $\mathrm{N}$-isopropylacrylamide gels. Macromolecules 23:365

11. Todd H, Robert $P$ (2008) Impact of microgel morphology on functionalized microgel-drug interactions. Langmuir 24:1005

12. You YZ, Kalebaila KK, Brock SL (2008) Temperature-controlled uptake and release in PNIPAM-modified porous silica nanoparticles. Chem Mater 20:3354

13. Wang Y, Zhang L, Tang C, Yu D (2015) Preparation and characterization of rubber-clay nanocomposites. J Appl Polym Sci 78:1879

14. Shi L, Yao J, Jiang J, Zhang L, Xu N (2009) Preparation of mesopore-rich carbons using attapulgite as templates and furfuryl alcohol as carbon source through a vapor deposition polymerization method. Microporous Mesoporous Mater 122:294

15. Peng L, Jiang L, Zhu L, Wang A (2014) Attapulgite/poly(acrylic acid) Nanocomposite (ATP/PAA) hydrogels with multifunctionalized attapulgite (org-ATP) nanorods as unique cross-linker: preparation optimization and selective adsorption of $\mathrm{Pb}$ (II) lon. Acs Sustain Chem Eng 2:269

16. Hua Z, Yong Z, Peng Z, Zhang Y (2010) Influence of the clay modification and compatibilizer on the structure and mechanical properties of ethylene\&ndash;propylene\&ndash;diene rubber/ montmorillonite composites. J Appl Polym Sci 92:638

17. Giussi JM, Von Bilderling C, Alarcón E et al (2018) Thermoresponsive PNIPAm nanopillars displaying amplified responsiveness through the incorporation of nanoparticles. Nanoscale 10:1189
18. Zhang H, Qin H, Li L, Zhou X, Wang W, Kan C (2017) Preparation and characterization of controlled-release avermectin/castor oilbased polyurethane nanoemulsions. J Agric Food Chem 66:6552

19. Gupta M, Shanker A (2008) Persistence of acetamiprid in tea and its transfer from made tea to infusion. Food Chem 111:805

20. Anirudhan TS, Ramachandran M (2008) Synthesis and characterization of amidoximated polyacrylonitrile/organobentonite composite for $\mathrm{Cu}(\mathrm{II}), \mathrm{Zn}(\mathrm{II})$, and $\mathrm{Cd}(\mathrm{II})$ adsorption from aqueous solutions and industry wastewaters. Ind Eng Chem Res 47:60

21. Googerdchian F, Moheb A, Emadi R (2012) Lead sorption properties of nanohydroxyapatite-alginate composite adsorbents. Chem Eng J 200-202:471

22. Liu B, Xia Y, Zhou Y, Nie W, Chen P (2017) Improved dispersion of attapulgite nanorods in polymer with graphene oxide nanosheets. J Mater Sci 52:1369

23. Langmuir I (1917) The constitution and fundamental properties of solids and liquids. J Franklin Inst 184:721

24. Mohamed MH, Wilson LD, Headley JV, Peru KM (2010) Sequestration of naphthenic acids from aqueous solution using $\beta$-cyclodextrin-based polyurethanes. Phys Chem Chem Phys 13:1112

25. Suárez M, Garcia E (2006) FTIR spectroscopic study of palygorskite: influence of the composition of the octahedral sheet. Appl Clay Sci 31:154

26. Wu N, Lei F, Ming S, Aslam M, Wong KC, Dravid VP (2015) Interaction of fatty acid monolayers with cobalt nanoparticles. Nano Lett 4:383

27. Mu B, Kang Y, Wang A (2013) Preparation of a polyelectrolytecoated magnetic attapulgite composite for the adsorption of precious metals. J Mater Chem A 1:4804

28. Xie R, Chu LY, Chen WM, Xiao W, Wang HD, Qu JB (2005) Characterization of microstructure of poly(n-isopropylacrylamide)grafted polycarbonate track-etched membranes prepared by plasma-graft pore-filling polymerization. J Membr Sci 258:157

29. Sanz B, Von BC, Tuninetti JS et al (2017) Thermally-induced softening of PNIPAm-based nanopillar arrays. Soft Matter 13:2453

30. Rawat P, Singh RN (2015) Synthesis and study on aroylhydrazones having cyanovinylpyrrole. Arab J Chem. https://doi. org/10.1016/j.arabjc.2015.03.001

31. Wang L, Sheng J (2005) Preparation and properties of polypropylene/org-attapulgite nanocomposites. Polymer 46:6243

32. Peng L, Wang T (2007) Preparation of well-defined star polymer from hyperbranched macroinitiator based attapulgite by surface-initiated atom transfer radical polymerization technique. Ind Eng Chem Res 46:97

33. Wu Z, Zhang X, Zhou C, Pang JL, Zhang PP (2017) Adsorption neutralization model and floc growth kinetics properties of aluminum coagulants based on sips and boltzmann equation. ACS Appl Mater Interfaces 9:5992

34. Cao-Luu N-H, Pham Q-T, Yao Z-H, Wang F-M, Chern C-S (2019) Synthesis and characterization of PNIPAM microgel core-silica shell particles. J Mater Sci 54:7503

Publisher's Note Springer Nature remains neutral with regard to jurisdictional claims in published maps and institutional affiliations. 